parameters. The final step in the demonstration of biosimilarity is a comparative phase 3 clinical trial in a representative indication

Objectives: The objective of this study was to evaluate the similarity of ABP 710 compared to RP with respect to efficacy and safety.

Methods: In this multicenter, randomized, double-blind study, the efficacy and safety of ABP 710 was compared with the RP in subjects with moderate to severe rheumatoid arthritis. Subjects were maintained on a stable 7.5 to $25 \mathrm{mg} /$ week dose of methotrexate and received $3 \mathrm{mg} / \mathrm{kg}$ infusions of the investigational products (IPs) at predetermined intervals. The primary endpoint of the study was risk difference (RD) of ACR20 at week 22; secondary endpoints included DAS28-CRP and safety at week 22. The confidence intervals $(\mathrm{Cl})$ for $\mathrm{RD}$ of ACR20 were estimated using the intention-to-treat analysis set with non-responder imputation based on the subject's randomized treatment. Clinical equivalence was evaluated by comparing the 2 -sided $90 \% \mathrm{Cl}$ with the equivalence margin of $(-15 \%$, $15 \%$ ) of the RD of ACR20. For change of DAS28-CRP at week 22 from baseline, the $\mathrm{Cl}$ of the mean difference was estimated using an analysis of covariance model with relevant baseline values and stratification factors as covariates.

Results: Of the 558 randomized subjects, 556 were treated (ABP $710 \mathrm{n}=278$; RP $n=278$ ). For ACR20, the RD was $9.3 \%$ and the lower bound of the $90 \%$ $\mathrm{Cl}(2.67 \%, 15.96 \%)$ was within the pre-specified criteria, thus confirming noninferiority; the upper bound slightly exceeded the pre-specified criteria such that superiority cannot be ruled out statistically. In post-hoc analysis when the impact of random imbalance in baseline demographic and disease characteristic between the 2 treatment arms was adjusted, the RD was reduced to $7.18 \%$ and the $90 \% \mathrm{Cl}$ was narrowed to $(0.75 \%, 13.62 \%)$. Results of change of DAS28-CRP at week 22 showed a difference in mean change from baseline of -0.01 with $90 \% \mathrm{Cl}$ of $(-0.20,0.17)$. Since this difference is less than the suggested EULAR threshold of 0.6 for clinically meaningful difference, the result supports both non-inferiority and non-superiority. Through week 22, percentage of subjects reporting any serious adverse events was 4.1 (ABP $710=3.2 ; \mathrm{RP}=5.0$ ) and the binding/neutralizing antibody positive post-baseline in subjects were 58.9/19.4 (ABP 710=57.1/18.0; RP=60.6/20.8).

Conclusion: The results of this study indicate that ABP 710 is similar to the RP. Although we were unable to statistically confirm non-superiority, post-hoc analysis is supportive of non-superiority. The DAS28-CRP difference of less than 0.6 is believed to not be clinically meaningful. These efficacy and safety results support similarity between ABP 710 and infliximab RP.

Acknowledgement: Sonya Lehto and Monica Ramchandani for medical writing. Study investigators and patients

Disclosure of Interests: Mark C. Genovese Grant/research support from: Sanofi/Genzyme, Genentech/Roche, RPharm, Consultant for: Sanofi/Genzyme, Genentech/Roche, RPharm, Juan Sanchez-Burson Speakers bureau: Lilly, Janssen, Pfizer, MyungShin Oh Shareholder of: Amgen, Employee of: Amgen, Éva Balázs Grant/research support from: Celltrion, Inc., Consultant for: Merck and Amgen, Jeffrey Neal Consultant for: Investigator Amgen, Gary Fanjiang Shareholder of: Amgen, Employee of:
Amgen, Stanley Cohen Grant/research support from: AbbVie, Amgen Inc., AstraZeneca, Biogen-IDEC, Bristol Meyer Squibb, Genentech, Janssen, Eli Lilly, Novartis, Pfizer, Merck, and Roche, Consultant for: Abbvie, Amgen, AstraZeneca, Biogen-IDEC, Bristol Meyer Squibb, Genentech, Janssen, Lilly, Novartis Pfizer, Merck and Roche

DOI: 10.1136/annrheumdis-2019-eular 4928

\section{AB0378 AN UPDATE AND SYSTEMATIC REVIEW ON USING BIOLOGICALS DURING PREGNANCY IN INFLAMMATORY AUTOIMMUNE DISEASES}

Nafise Ghalandari ${ }^{1,2,3}$, Radboud Dolhain ${ }^{1,3},{ }^{1,2}$ Johanna Hazes $^{3}$, Eugène van Puijenbroek ${ }^{4}$, Manavi Kapur ${ }^{2}$, Hubertina Johanna Maria Josephina Crijns ${ }^{2}$ ${ }^{1}$ Erasmus MC, Academic Center of Inflammunity, Rotterdam, Netherlands; ${ }^{2}$ Dutch Medicines Evaluation Board (CBG-MEB), Utrecht, Netherlands; ${ }^{3}$ Erasmus MC, Rheumatology, Rotterdam, Netherlands; ${ }^{4}$ Netherlands Pharmacovigilance Center Lareb, 's Hertochenbosch, Netherlands

Background: The effects of biologicals on reproduction are unknown Therefore pregnancy during biologicals use is discouraged. However biologicals are frequently prescribed in patients of childbearing potential and many patients become pregnant while using these drugs.

Objectives: In 2015 EULAR published a broad review article on this matter. Our aim was to update the data based on newly published articles. Methods: A search was conducted at 18/10/2017 and then 21/11/2018 in Embase, Medline Ovid, Web Of Science, Cochrane CENTRAL and Google Scholar with specific search terms for each database. Articles were excluded based on title/abstract (double blind, two researchers, NG and HJMJC) and then full text (one researcher, NG). A chart was made based on outcomes of interest(see Table1). The references of included articles were reviewed to include and minimize the missing articles.

Results: Totally 137 articles were included (79 articles at first round, 51 articles at second round and 7 found after screening the references of the included articles). A descriptive analysis was performed to sum up the data on each separate biological and all the biologicals together. The results are shown in Table1. It should be notified that due to heterogeneity of the data the calculated percentages are up to individual interpretations and cannot be compared to each other.

Conclusion: From 2015 onwards, there have been many articles published regarding use of biologicals during pregnancy. Despite limitations of our study, such as heterogeneity in being prospective or retrospective and quality of the included articles, no alarm is raised concerning safety issues.

Disclosure of Interests: Nafise Ghalandari: None declared, Radboud Dolhain Grant/research support from: UCB Pharma B.V, Johanna Hazes: None declared, Eugène van Puijenbroek: None declared, Manavi Kapur: None declared, Hubertina Johanna Maria Josephina Crijns: None declared DOI: 10.1136/annrheumdis-2019-eular.7108

Table 1. Results of the final chart. The denominator is the sum up of total cases recruited from the articles, for each outcome the denominator is defined as the population for which the specific outcome was reported. The other cases, which haven't mentioned the outcome in their results, were excluded from the denominator for that specific outcome. Both spontaneous and elective abortion cases are excluded from the denominator of preterm birth and major congenital malformations.

\begin{tabular}{|c|c|c|c|c|c|c|c|c|}
\hline Drug & $\begin{array}{l}\text { Maternal exposed } \\
\text { pregnancies }\end{array}$ & Elective & $\begin{array}{l}\text { ortion } \\
\text { Spontaneous }\end{array}$ & Live birth & $\begin{array}{l}\text { Preterm } \\
\text { birth }\end{array}$ & $\begin{array}{c}\text { Major congenital } \\
\text { malformations }\end{array}$ & $\begin{array}{l}\text { Serious infections in } \\
\text { children }\end{array}$ & $\begin{array}{l}\text { ADRs to } \\
\text { vaccine }\end{array}$ \\
\hline Abatacept & 153 & $\begin{array}{c}13.1 \% \\
(20 / 153)\end{array}$ & $\begin{array}{c}26.1 \% \\
(40 / 153)\end{array}$ & $\begin{array}{c}60.8 \% \\
(93 / 153)\end{array}$ & - & $\begin{array}{l}6.8 \% \\
(6 / 88)\end{array}$ & - & $\begin{array}{l}0.0 \% \\
(0 / 93)\end{array}$ \\
\hline Adalimumab & 2518 & $\begin{array}{c}0.1 \% \\
(3 / 339)\end{array}$ & $\begin{array}{c}0.4 \% \\
(16 / 432)\end{array}$ & $\begin{array}{c}95.5 \% \\
(424 / 444)\end{array}$ & $\begin{array}{c}3.2 \% \\
(7 / 213)\end{array}$ & $\begin{array}{c}3.7 \% \\
(23 / 608)\end{array}$ & $\begin{array}{c}7.9 \% \\
(4 / 501)\end{array}$ & $\begin{array}{r}0.5 \% \\
\left(1 / 205^{*}\right)\end{array}$ \\
\hline Anakinra & 34 & $\begin{array}{l}2.9 \% \\
(1 / 34)\end{array}$ & $\begin{array}{l}2.9 \% \\
(1 / 34)\end{array}$ & $\begin{array}{l}94.2 \% \\
(32 / 34)\end{array}$ & $\begin{array}{l}20.0 \% \\
(2 / 10)\end{array}$ & $\begin{array}{r}3.4 \% \\
\left(1 / 29^{\dagger}\right)\end{array}$ & $\begin{array}{l}0.0 \% \\
(0 / 30)\end{array}$ & - \\
\hline $\begin{array}{l}\text { Certolizumab } \\
\text { pegol }\end{array}$ & 767 & $\begin{array}{c}4.7 \% \\
(27 / 574)\end{array}$ & $\begin{array}{c}8.2 \% \\
(47 / 574)\end{array}$ & $\begin{array}{c}86.9 \% \\
(527 / 606)\end{array}$ & $\begin{array}{c}10.7 \% \\
(41 / 380)\end{array}$ & $\begin{array}{c}1.9 \% \\
(10 / 510)\end{array}$ & $\begin{array}{l}4.0 \% \\
(2 / 50)\end{array}$ & $\begin{array}{l}0.0 \% \\
(0 / 26)\end{array}$ \\
\hline Etanercept & 801 & $\begin{array}{c}7.6 \% \\
(30 / 393)\end{array}$ & $\begin{array}{c}17.4 \% \\
(69 / 397)\end{array}$ & $\begin{array}{c}87.4 \% \\
(702 / 803)\end{array}$ & $\begin{array}{c}11.5 \% \\
(40 / 347)\end{array}$ & $\begin{array}{c}6.2 \% \\
(37 / 597)\end{array}$ & $\begin{array}{c}0.0 \% \\
(0 / 140)\end{array}$ & $\begin{array}{c}0.4 \% \\
(1 / 269)\end{array}$ \\
\hline Infliximab & 2174 & $\begin{array}{c}5.1 \% \\
(90 / \\
1759)\end{array}$ & $\begin{array}{c}9.6 \% \\
(169 / 1759)\end{array}$ & $\begin{array}{c}79.3 \% \\
(1483 / \\
1869)\end{array}$ & $\begin{array}{c}5.9 \% \\
(111 / 1892)\end{array}$ & $\begin{array}{c}10.9 \% \\
(22 / 2014)\end{array}$ & $\begin{array}{c}13.7 \% \\
(21 / 153)\end{array}$ & $\begin{array}{l}18.2 \% \\
(6 / 33)\end{array}$ \\
\hline Rituximab & 27 & $\begin{array}{l}0.0 \% \\
(0 / 11)\end{array}$ & $\begin{array}{l}12.5 \% \\
(2 / 16)\end{array}$ & $\begin{array}{l}87.5 \% \\
(14 / 16)\end{array}$ & $\begin{array}{l}18.2 \% \\
(2 / 11)\end{array}$ & $\begin{array}{l}0.0 \% \\
(0 / 8)\end{array}$ & $\begin{array}{l}0.0 \% \\
(0 / 18)\end{array}$ & - \\
\hline Tocilizumab & 368 & $\begin{array}{c}14.6 \% \\
(37 / 254)\end{array}$ & $\begin{array}{c}20.9 \% \\
(53 / 254)\end{array}$ & $\begin{array}{c}64.2 \% \\
(163 / 254)\end{array}$ & $\begin{array}{c}18.1 \% \\
(32 / 177)\end{array}$ & $\begin{array}{c}4.9 \% \\
(8 / 164)\end{array}$ & $\begin{array}{l}0.0 \% \\
(0 / 3)\end{array}$ & - \\
\hline Tofacitinib ${ }^{\varepsilon}$ & 47 & $\begin{array}{l}19.5 \% \\
(8 / 41)\end{array}$ & $\begin{array}{l}17.1 \% \\
(7 / 41)\end{array}$ & $\begin{array}{l}63.4 \% \\
(26 / 41)\end{array}$ & $\begin{array}{l}7.7 \% \\
(2 / 26)\end{array}$ & $\begin{array}{l}3.8 \% \\
(1 / 26)\end{array}$ & $\begin{array}{l}0.0 \% \\
(0 / 26)\end{array}$ & - \\
\hline Ustekinumab & 46 & $\begin{array}{l}0.0 \% \\
(0 / 28)\end{array}$ & $\begin{array}{l}7.1 \% \\
(2 / 28)\end{array}$ & $\begin{array}{l}92.6 \% \\
(25 / 27)\end{array}$ & $\begin{array}{l}3.8 \% \\
(1 / 26)\end{array}$ & $\begin{array}{l}0.0 \% \\
(0 / 21)\end{array}$ & $\begin{array}{l}0.0 \% \\
(0 / 10)\end{array}$ & $\begin{array}{l}0.0 \% \\
(0 / 3)\end{array}$ \\
\hline Vedolizumab & 59 & $\begin{array}{l}11.1 \% \\
(6 / 54)\end{array}$ & $\begin{array}{l}18.5 \% \\
(10 / 54)\end{array}$ & $\begin{array}{l}70.4 \% \\
(38 / 54)\end{array}$ & $\begin{array}{l}2.9 \% \\
(1 / 34)\end{array}$ & $\begin{array}{l}10.0 \% \\
(2 / 20)\end{array}$ & $\begin{array}{l}0.0 \% \\
(0 / 28)\end{array}$ & - \\
\hline
\end{tabular}

